DIVISION OF THE HUMANITIES AND SOCIAL SCIENCES

CALIFORNIA INSTITUTE OF TECHNOLOGY

PASADENA, CALIFORNIA 91125

IMPLICATIONS OF PARETO EFFICIENCY FOR TWO-AGENT (HOUSEHOLD) CHOICE

Federico Echenique and Lozan Ivanov

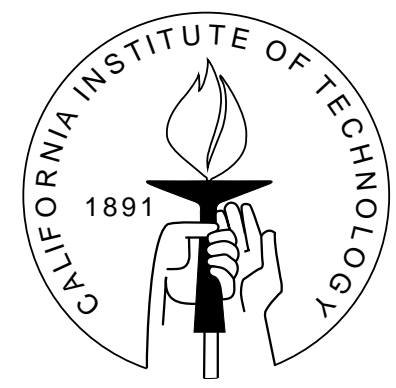

SOCIAL SCIENCE WORKING PAPER 1308

July 2009 


\title{
Implications of Pareto Efficiency for Two-Agent (Household) Choice
}

\author{
Federico Echenique and Lozan Ivanov
}

\begin{abstract}
We study when two-member household choice behavior is compatible with Pareto optimality. We ask when an external observer of household choices, who does not know the individuals' preferences, can rationalize the choices as being Pareto-optimal. Our main contribution is to reduce the problem of rationalization to a graph-coloring problem. As a result, we obtain simple tests for Pareto optimal choice behavior. In addition to the tests, and using our graph-theoretic representation, we show that Pareto rationalization is equivalent to a system of quadratic equations being solvable.
\end{abstract}

JEL classification numbers: D10,D13

Key words: Revealed preference; Pareto optimality; Testable implications. 


\title{
Implications of Pareto Efficiency for Two-Agent (Household) Choice*
}

\author{
Federico Echenique and Lozan Ivanov
}

\section{Introduction}

We study the implications of efficiency for choice behavior. Concretely, for a given collective of agents, we want to describe the choices that are consistent with Pareto optimality, when we are ignorant about the individual agents' preferences. We develop a series of simple necessary conditions (tests) for choice to be consistent with efficiency; the tests lead up to a necessary and sufficient condition.

Consider a household (a two-person collective) that has to select an alternative from a finite set. For instance, a couple may have to decide how to spend their income, or two researchers who work at the same laboratory may have to choose which projects to fund with their common grants.

Suppose we are given the households' choice behavior. That is, we know what the household would choose from each possible subset of alternatives. We ask when the observed choice can be Pareto rationalized: we want to know when we can find two preference relations, one for each household member, such that for any given subset of alternatives, the choices are exactly the Pareto optimal alternatives within the set. We are interested in what the choice behavior of such a household looks like, and how we can test if a given household's choices can be rationalized.

Pareto rationalization depends on certain conditions that the collective choices must satisfy. Some conditions are classical: For example, if an alternative $x$ is selected from a set $A$, and we shrink $A$ to $B \subseteq A$ while maintaining $x$ as still available in $B$, then $x$ must be chosen from $B$; this condition is usually called Chernoff's Axiom. The classical conditions allow us to work with two binary relations: revealed Pareto domination $R$ and Pareto indifference $O$. Pareto rationalization requires that there be two individual preferences $>_{1}$ and $>_{2}$ such that $x R y$ if and only if $x$ Pareto dominates $y$, and $x O y$ if

${ }^{*}$ We thank Leeat Yariv for comments on an earlier draft. Our research was supported by the Lee Center at Caltech. 
and only if $x$ and $y$ are Pareto incomparable. We present new necessary and sufficient conditions for Pareto rationalization.

The problem is motivated by revealed preference theory. In revealed preference theory, one wants to know when the choice behavior of an individual agent is rational. By observing his choice from each pair of alternatives, we can gain complete knowledge of the agent's preferences and then judge whether his behavior is rational or not according to some external criterion (e.g. whether his preferences are acyclic, transitive, etc.)

We consider instead the collective behavior of two agents. Their choices reveal their collective preferences, but we cannot reconstruct their individual preferences, and therefore cannot outright judge whether they are rational or not. To make it harder, the observed group preferences, $R$ and $O$, need not satisfy some classical rationality conditions such as acyclicity.

We present the paper in terms of rationalizing choice, but one can also phrase the results as a contribution to dimension theory: A partial order $\succ$ on a set $X$ has dimension $N$ if there are $n$ linear orders $>_{i}, i=1, \ldots n$ such that

$$
x \succ y \Leftrightarrow \forall i\left(x>_{i} y\right)
$$

We focus on the case $n=2$. The problem was first studied by Dushnik and Miller (1941), who provide a characterization of the two-agent rationalizable orders. The problems of dimensions $n>2$ are open. ${ }^{1}$ The Dushnik-Miller result is a deep result about partial orders, but it does not constitute a useful test for rationalizability because it is nonconstructive. The characterization is in terms of a property of the partial order that cannot be verified constructively.

In the economic literature, Sprumont (2001) has studied two-agent Pareto rationalizability when one is interested in preferences with a particular structure. ${ }^{2}$ Sprumont works with a continuum of alternatives, and studies Pareto two-agent rationalizations with "regular" preferences. We instead focus on the basic discrete case, where we put no structure on agents' preferences. We shall work with strict preferences in the main discussion of our results, but in the final section of the paper we show that household choices are rationalizable in strict preferences if and only if they are rationalizable in nonstrict preferences.

The following observation is key in our analysis. Consider a pair of alternatives, $(x, y)$. Then one of two things can happen: either one alternative is revealed Pareto preferred to the other (say $x R y$ if $x$ is chosen out of the set $\{x, y\}$ ) or the two alternatives are not Pareto ranked $(x O y)$. The first case is simple: there is no ambiguity as any rationalizing preferences must coincide with the Pareto order; if $x R y$ and $>_{1},>_{2}$ are rationalizing preferences, then $x>_{1} y$ and $x>_{2} y$. The second case presents us with a choice. If $x O y$

\footnotetext{
${ }^{1}$ Dimension theory was introduced by Dushnik and Miller in their 1941 article. A large literature on dimension theory has been developed: the book by Trotter (2001) is a recent exposition.

${ }^{2}$ See also Sprumont (2000), who works out the relationship between Pareto and Nash rationalizability.
} 
then either $x>_{1} y$ and $y>_{2} x$ or $y>_{1} x$ and $x>_{2} y$. In the first case, the direction of individual preference is determinate. In the second case we have a degree of freedom and we cannot infer individual preferences from the Pareto ranking. This degree of freedom makes the problem of Pareto rationalization substantially different from classical revealed preference theory.

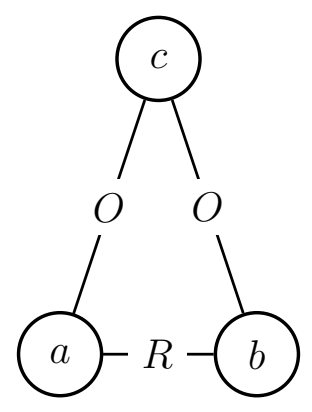

Figure 1: $(a c, b c): a R b$ or $b R a, a O c$ and $c O b$.

We represent the problem in graph theoretic terms. We think of each alternative as a vertex in a complete graph. For any two alternatives $x$ and $y$, the edge $x y$ is labeled with $R$ or $O$. The key building block of our analysis is in Figure 1, where $a R b, a O c$ and $c O b$. It is easy to see that if $\left(>_{1},>_{2}\right)$ is a rationalization then either $c$ is the best or the worst alternative for $>_{1}$ out of the set $\{a, b, c\}$. Furthermore, if $c$ is the best for $>_{1}$, then it is the worst for $>_{2}$, and vice versa. So one agent's preference points towards $c$ and other's away from $c$.

A graph contains (in principle) many configurations like the one in Figure 1. If we are trying to build a rationalization $\left(>_{1},>_{2}\right)$ then the decision we make on one such configuration, in terms of whether agent 1 or agent 2 prefers $c$, affects the decision we make on others. We reduce this problem to one of graph bi-coloring: one color for the triplet $(a c, b c)$ represents agent 1 preferring $c$ out of $\{a, b, c\}$; the other color represents agent 2 preferring $c$ out of $\{a, b, c\}$.

This graph coloring approach allows us to formulate a simple test (a necessary condition) for Pareto rationalizability. The test is based on the two-coloring rationality condition being equivalent to the absence of certain odd cycles; this test is easy to implement. Passing the test is, unfortunately, not sufficient to guarantee Pareto rationalizability. We present another necessary condition for choice behavior to be Pareto rationalizable. The condition is based on solving a particular quadratic system of equations where each variable can only take the values 1 and -1 . This condition, together with the test and two classical revealed preference axioms, is sufficient for choice behavior to be Pareto rationalizable.

The rest of the paper is organized as follows. Section 2 presents some preliminary results. In Section 3 we present the graph-theoretic notions we employ. Section 4 contains our main results. We remark on the extension of our results to non-strict preferences in Section 5. We present some conclusions in Section 6 . 


\section{Preliminaries}

\subsection{Definitions}

Let $X$ be a nonempty, finite set of alternatives. We call a nonempty subset $B \subseteq X$ a budget. Denote by $\mathcal{P}(X)$ the set of all budgets $B \subseteq X$. A choice function is a function

$$
g: \mathcal{P}(X) \rightarrow \mathcal{P}(X)
$$

such that $g(B) \subseteq B$ for all $B \in \mathcal{P}(X)$.

A strict preference relation on $X$ is a total, antisymmetric and transitive binary relation on $X$ (a linear order).

Given two preference relations, $>_{1}$ and $>_{2}$, and a budget $B \in \mathcal{P}(X)$, say that $a \in B$ is Pareto dominated in $B$ with respect to $>_{1}$ and $>_{2}$ if there is some $b \in B$ such that $b>_{1} a$ and $b>_{2} a$; in this case we say that $b$ Pareto dominates $a$ (with respect to the two preferences $>_{1}$ and $>_{2}$ ). We call $a \in B$ Pareto efficient in $B$ if it is not Pareto dominated in $B$. Observe that if $a$ and $b$ are Pareto efficient in some set $B \supseteq\{a, b\}$, then either $a>_{1} b$ and $b>_{2} a$, or $b>_{1} a$ and $a>_{2} b$

A choice function $g$ is two-agent Pareto rationalizable if there are two strict preference relations $>_{1}$ and $>_{2}$, such that for all $B \in \mathcal{P}(X), f(B)$ is the set of all Pareto efficient alternatives in $B$ with respect to $>_{1}$ and $>_{2}$. In this case, we say that $\left(>_{1},>_{2}\right)$ is a Pareto rationalization of $f$, or a rationalizing pair.

A pair of sets $(V, E)$ is a (directed) graph whenever $E \subseteq V \times V$. We say that the elements of $V$ are the vertexes of the graph, and that $\left(v, v^{\prime}\right) \in E$ means that there is an edge pointing from $v$ to $v^{\prime}$.

\subsection{Preliminary results}

We present two axioms that are necessary for Pareto rationalization. They are standard axioms in the literature, and they are known to be necessary for single-agent rationalization as well (see e.g. Moulin (1991)). The results in Lemmas (1) and (2) are also standard.

Axiom 1. For all $B_{1}, B_{2} \in \mathcal{P}(X)$, if $B_{1} \subseteq B_{2}$ and $a \in B_{1} \cap f\left(B_{2}\right)$, then $a \in f\left(B_{1}\right)$.

The interpretation of Axiom 1 is that if $f$ is Pareto rationalizable, and $a$ is a Pareto efficient choice among the alternatives in a given budget, it must remain Pareto efficient among the alternatives of any smaller budget that contains $a$. Axiom 1 is a standard axiom in choice theory, usually called Chernoff's axiom (Moulin (1991)). We obtain the following version of this standard result

Lemma 1. Axiom 1 is necessary for Pareto rationalizability. 
Proof. Suppose that $f$ is Pareto rationalizable and that $\left(>_{1},>_{2}\right)$ is a rationalization. By definition, we have that if $a \in B_{1} \cap f\left(B_{2}\right)$ for some $B_{1} \subseteq B_{2}$, then $a$ is not dominated by any $b \in B_{2}$ (with respect to $\left(>_{1},>_{2}\right)$ ). Since $B_{1} \subseteq B_{2}$ it follows that $a$ is not dominated by any $b \in B_{1}$. Thus, $a$ is Pareto efficient in $B_{1}$ and $a \in f\left(B_{1}\right)$, as $f\left(B_{1}\right)$ is the set of all Pareto efficient alternatives in $B_{1}$ with respect to the rationalization $\left(>_{1},>_{2}\right)$. This proves the lemma.

Axiom 2. For all $B_{1} \in \mathcal{P}(X)$, if $a \in B_{1} \backslash f\left(B_{1}\right)$, then for some $b \in f\left(B_{1}\right)$

$$
f(a b)=b .^{3}
$$

The interpretation of Axiom 2 is that if $f$ is Pareto rationalizable, and $a$ is not Pareto efficient among the alternatives in a given budget, then there must exist an efficient alternative in this budget which Pareto dominates it. The following lemma is essentially in Moulin (1991), page 306:

Lemma 2. Axiom 2 is necessary for Pareto rationalizability.

Proof. Suppose that $\left(>_{1},>_{2}\right)$ is a rationalization and $a \in B_{1} \backslash f\left(B_{1}\right)$ for some $B_{1} \in \mathcal{P}(X)$. Then $a$ is not Pareto efficient in $B_{1}$ with respect to $\left(>_{1},>_{2}\right)$. If follows that $a$ is Pareto dominated in $B_{1}$ by some other alternative $a_{1} \in B_{1}$. If $a_{1} \notin f\left(B_{1}\right)$ then $a_{1} \in B_{1} \backslash f\left(B_{1}\right)$ and by the same argument $a_{1}$ is Pareto dominated in $B_{1}$ by some other alternative $a_{2} \in B_{1}$. And so on, since the set $B_{1}$ is finite and rationalizing preferences are transitive, we will eventually find an alternative $b \in f\left(B_{1}\right)$ that Pareto dominates $a$, and hence $b$ is the only Pareto efficient alternative in the budget $a b: f(a b)=b, b \in f\left(B_{1}\right)$.

We next introduce two binary relations. Given is a choice function $f$. The strict revealed preference relation associated to $f$ is the binary relation $R$ defined as $a R b$ if $f(a b)=a{ }^{4}$ The indifference relation associated to $f$ is the binary relation $O$ defined as $a O b$ and $b O a$ if $f(a b)=a b$. Note that $O$ is symmetric.

The following well-known (Moulin, 1991) observation illustrates the importance of $R$. Lemma 3. If $f$ satisfies Axioms 1 and $2, R$ is transitive.

Proof. Assume that $f$ satisfies both axioms and $a R b, b R c$. Using Axiom 1 twice we get $b \notin f(a b) \Rightarrow b \notin f(a b c)$ and $c \notin f(b c) \Rightarrow c \notin f(a b c)$. Since $f(a b c)$ is nonempty it follows that $f(a b c)=a$. Now Axiom 2 implies that $f(a c)=a$ as $a$ is the only alternative in $f(a b c)$. Thus $a R c$ and the relation $R$ is transitive.

Remark. Note that $O$ may not be transitive, as the following example with $X=\{a, b, c\}$ illustrates:

$$
\begin{aligned}
& c>_{1} a>_{1} b, \\
& b>_{2} c>_{2} a ;
\end{aligned}
$$

where $a O b$ and $b O c$ but $c R a$.

\footnotetext{
${ }^{3} \mathrm{We}$ are going to abuse notation and write $a_{1} a_{2} \ldots a_{t}$ instead of $\left\{a_{1}, a_{2}, \ldots, a_{t}\right\}$.

${ }^{4}$ The relation $R$ is also called the base relation.
} 
We henceforward assume that the choice function $f$ satisfies Axioms 1, 2 and so $R$ is transitive. We are interested in when a pair of linear orders $\left(>_{1},>_{2}\right)$ is a rationalization of the choice function $g$, given that it satisfies both axioms. Suppose first that $\left(>_{1},>_{2}\right)$ is a rationalization. Note $f(a b)$ is either a singleton or $f(a b)=a b$. If $f(a b)=a$ is a singleton then $a>_{1} b$ and $a>_{2} b$ as $a$ is the only efficient element in $a b$ with respect to $\left(>_{1},>_{2}\right)$. If $f(a b)=a b$, then both $a$ and $b$ must be efficient, meaning that

$$
\left(\left(a>_{1} b\right) \text { and }\left(b>_{2} a\right)\right) \text { or }\left(\left(a>_{2} b\right) \text { and }\left(b>_{1} a\right)\right) \text {. }
$$

We arrive at the following important observation.

Lemma 4. Suppose the choice function $f$ satisfies Axioms 1 and 2. Then a pair of binary relations $\left(>_{1},>_{2}\right)$, over the universal set $X$, is a rationalization of $f$ if and only if $a>_{1} b$ and $a>_{2} b$ whenever $a R b$, (1) holds whenever $a O b$, and both relations are acyclic.

Proof. We already proved the forward direction of the claim. Conversely, suppose that $\left(>_{1},>_{2}\right)$ is a pair of acyclic relations such that $a>_{1} b$ and $a>_{2} b$ whenever $a R b$, and (1) holds whenever $a O b$. By these assumptions, $>_{1}$ and $>_{2}$ are total and antisymmetric as well. Since both relations are acyclic, it follows that they must also be transitive and hence linear orders.

Consider a budget $B \in \mathcal{P}(X)$ and an alternative $b \in B \backslash f(B)$. By Axiom $2, f(a b)=a$ for some $a \in f(B)$. That is, $a R b$. Then, by assumption, $a>_{1} b$ and $a>_{2} b$, and so $b$ is Pareto dominated in $B$ with respect to the pair $\left(>_{1},>_{2}\right)$. On the other hand, take any $a \in f(B)$ and suppose that for some $c \in B, c>_{1} a$ and $c>_{2} a$. Since both relations agree over the pair of alternatives $(a, c)$, our assumptions imply that $c R a$. That is, $a \notin f(a c)$. But $a \in f(B) \cap\{a, c\}$ and by Axiom $1 a \in f(a c)$, a contradiction. Thus, $c>_{1} a$ and $c>_{2} a$ cannot simultaneously hold for any $c \in B$, which implies that $a$ is not Pareto dominated in $B$, i.e., $a$ is Pareto efficient in $B$ with respect to the pair of relations $\left(>_{1},>_{2}\right)$. Since $a \in f(B)$ and $b \in B \backslash f(B)$ are generic elements of the corresponding sets, we conclude that $f(B)$ contains the Pareto efficient alternatives in $B$ and only those. Hence, $\left(>_{1},>_{2}\right)$ is a Pareto rationalization of the choice function $f$.

Axioms 1 and 2 are necessary. We present an example below to the effect that they are not sufficient. By Lemma 4, then, it should be clear that property (1) captures the restrictions in Pareto rationalizability, in addition to the standard properties in Axioms 1 and 2. In the next section we shall use a graph-theoretic structure to understand property (1) better. 


\section{$3 \quad$ A graph coloring problem}

We proceed to illustrate how we translate the problem of characterizing Pareto rationalizability into a graph-coloring problem. Suppose that $\left(>_{1},>_{2}\right)$ is a rationalization of the choice function $f$. By Lemma 4 , we know this is equivalent to $\left(>_{1},>_{2}\right)$ being a pair of acyclic relations such that $a>_{1} b$ and $a>_{2} b$ whenever $a R b$, and (1) holds whenever $a O b$. We can rewrite these two properties of $\left(>_{1},>_{2}\right)$ as

$$
\text { if } a>_{1} b \text { and } a R b \text {, then } a>_{2} b \text {; if } a>_{1} b \text { and } a O b \text {, then } b>_{2} a \text {. }
$$

As a consequence, a rationalizing relation $>_{1}$ defines a rationalization $\left(>_{1},>_{2}\right)$. A choice function $g$ defines $R$ and $O$; so $>_{1}$ and (2) gives us $>_{2}$. This simple observation will allow us to translate characterizing Pareto rationalizability into a graph-coloring problem.

Let $G$ be the directed graph

$$
G=(X,\{a b \mid a R b \text { or } a O b\}) .
$$

The vertices of $G$ are all the alternatives from the universal set $X$ and the edges of $G$ represent all the revealed binary comparisons of alternatives in $X$ : we label the edge $(a, b)$ by $R$ if $a R b$ or $b R a$, and we label the edge $(a, b)$ by $O$ if $a O b$ or $b O a$. For example, imagine three alternatives $a, b$ and $c$ for which $a O c, c O b$ and $a R b$. The situation is represented in Figure 1 in the Introduction.

The configuration in Figure 1 is crucial. This configuration would not be possible if $R$ described a single agent's strict preference relation, and $O$ described her indifference relation. With a Pareto rationalization $\left(>_{1},>_{2}\right)$, we see that $>_{1}$ must either point away from c or point towards $c$ :

Lemma 5. Suppose that $\left(>_{1},>_{2}\right)$ is a rationalization and $a, b, c \in X$ are three alternatives for which $a O c, c O b$, and $a R b$ or $b R a$. Then the following is true

$$
\left(a>_{1} c \text { and } b>_{1} c\right) \text { or }\left(c>_{1} a \text { and } c>_{1} b\right) .
$$

Proof. Let $a R b$. We cannot have $b>_{1} c$ and $c>_{1} a$, as this results in the cycle $a>_{1} b>_{1} c>_{1} a$, contradicting the assumption that $>_{1}$ is acyclic (we have $a>_{1} b$ and

$a>_{2} b$ since $\left.a R b\right)$. Similarly, we cannot have $a>_{1} c$ and $c>_{1} b$ because this implies $b>_{2} c$ and $c>_{2} a$, which contradicts the acyclicity of $>_{2}$. Two possibilities remain: either $a>_{1} c$ and $b>_{1} c$, or $c>_{1} a$ and $c>_{1} b$. That is, (3) holds. We readily see that, after relabeling $a$ and $b$, (3) also holds if we have $b R a$ instead of $a R b$.

Remark. Statement (3) in Lemma 5 clearly holds for $>_{2}$ as well; and if one of the alternatives in (3) holds for $>_{1}$, the other holds for $>_{2}$. 
Given that (3) holds for any rationalization $\left(>_{1},>_{2}\right)$ and any three alternatives $a, b, c \in X$ with $a O c, c O b$, and $a R b$ or $b R a$, we seek to understand the structure of the graph $G$. Any necessary condition for $G$ that we hope to derive is indirectly a condition for the choice function $f$, as $G$ depends on the revealed preference relations $R$ and $O$, which, in turn, correspond to choice functions satisfying Axioms 1 and 2 .

We can express our observation (3) using a second graph, which is undirected:

$$
F=(\{a b \mid a \neq b\},\{(a c, b c) \mid a R b, a O c, b O c\})
$$

Note that the vertexes of $F$ are edges of $G$; there is an edge between $a c$ and $b c$ if they are in a relation like the one in Figure 1: they are elements of $O$ which are related by $R$.

We say that the edge $f=(a c, b c) \in F$ is colored 1 if $\left(a>_{1} c\right.$ and $\left.b>_{1} c\right)$, and that $f$ is colored -1 if $\left(c>_{1} a\right.$ and $\left.c>_{1} b\right)$. Consider Figure 1: the edge $(a c, b c)$ is colored 1 when $>_{1}$ points away from the common vertex $c$; the edge is colored -1 when $>_{1}$ points toward the common vertex. Since the assumed rationalization $\left(>_{1},>_{2}\right)$ verifies equation (3) by Lemma 5, it follows that it induces a coloring of every edge of the graph $F$. We now obtain a simple necessary condition for rationalizability based on this coloring.

Consider two adjacent edges $f_{1}, f_{2} \in F$; let $f_{1}=(a c, b c)$, for alternatives $a, b$, and $c$ such that the vertex $b c$ of $F$ is an endpoint of the edge $f_{2}$. We have $f_{2}=(c b, d b)$ or $f_{2}=(b c, d c)$ for some other alternative $d$. The first possibility is represented on the left in Figure 2 while the second possibility is on the right. When two adjacent edges $f_{1}=(a c, b c), f_{2}=(c b, d b)$ are related as in Figure 2 on the left, we say that they are dichromatic as they must have different colors in any rationalization. When $f_{1}=(a c, b c), f_{2}=(b c, d c)$ are related as in Figure 2 on the right, we say they are monochromatic as they must have the same color.

Formally, we say that a pair of edges $\left(f_{1}, f_{2}\right)$, of the graph $F$ is monochromatic when $f_{1}=(a c, b c)$ and $f_{2}=(b c, d c)$ for distinct alternatives $a, b, c, d \in X$. When $f_{1}=(a c, b c)$ and $f_{2}=(c b, d b)$ for distinct alternatives $a, b, c, d \in X$, we call the pair of edges $\left(f_{1}, f_{2}\right)$ dichromatic.

Lemma 6. Let $\left(>_{1},>_{2}\right)$ be a rationalization and $f_{1}, f_{2}$ be two adjacent edges in the graph $F$. If they are related as on the left in Figure 2 then the two edges have different colors. If $f_{1}, f_{2}$ are related as on the right, they have the same color.

Proof. Consider first the case $f_{1}=(a c, b c), f_{2}=(c b, d b)$. If the edge $f_{1}$ is colored -1 then $c>_{1} b$, and by equation (3) $d>_{1} b$ so that $f_{2}$ is colored 1. Similarly, if $f_{1}$ is colored 1 then $b>_{1} c$, and by $(3) b>_{1} d$ : $f_{2}$ is colored -1 . Either way, $f_{1}$ and $f_{2}$ have different colors. The second case $f_{1}=(a c, b c), f_{2}=(b c, d c)$ is treated the same way.

These ideas allow us to formulate a simple condition.

Axiom 3. Every cycle in $F$ has an even number of dichromatic pairs. 

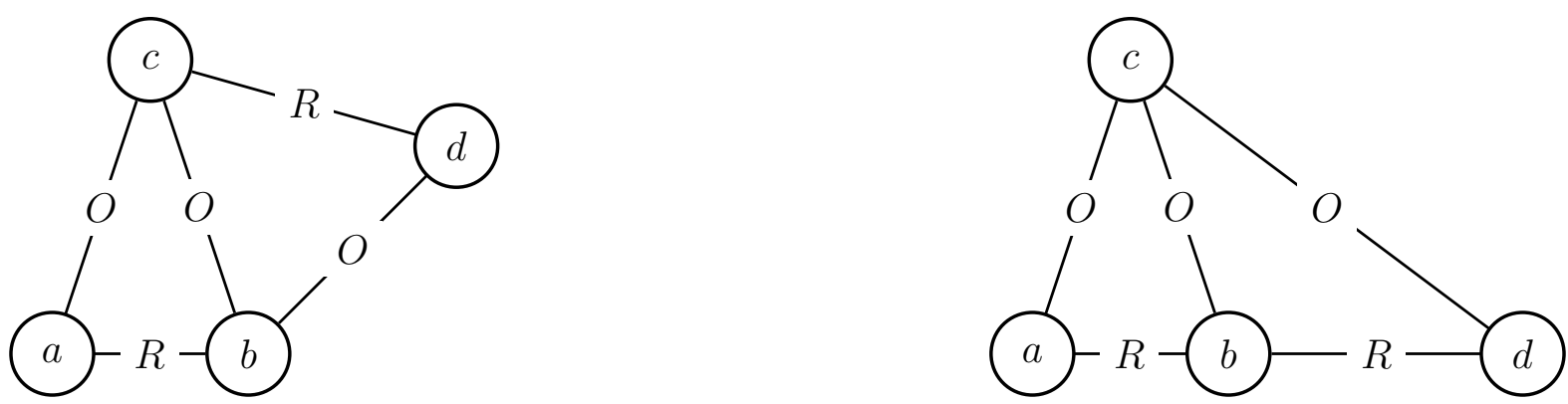

Figure 2: Left: $(a c, b c)$ and $(c b, d b)$ are dichromatic; right: $(a c, b c)$ and $(b c, c d)$ are monochromatic

We have argued that a rationalization implies a coloring of the edges of $F$ in which monochromatic pairs have the same color, and dichromatic pairs have opposite colors. Coloring using two colors (bicoloring) is equivalent to the absence of a cycle in which there is an odd number of vertexes forcing a switch in the color of adjacent edges (a simple result in graph theory). In our particular case, the vertex linking two adjacent edges of the graph $F$ forces a switch in color if they are dichromatic and forces them to have the same color if they are monochromatic. We get for free the following

Lemma 7. If $f$ is Pareto rationalizable, it must satisfy Axiom 3.

We finish this section with an example of a choice function that satisfies Axioms 1 and 2, but not 3. Such a function is not two-agent Pareto rationalizable.

Example. Let $X=\left\{a_{1}, a_{2}, a_{3}, a_{4}, a_{5}, a_{6}\right\}$ be the set of alternatives and define the choice function $g$ by the following table; the entry corresponding to row $a_{i}$ and column $a_{j}$ is $g\left(\left\{a_{i}, a_{j}\right\}\right)$.

$\begin{array}{ccccccc} & a_{1} & a_{2} & a_{3} & a_{4} & a_{5} & a_{6} \\ a_{1} & & a_{1} & a_{3} a_{1} & a_{1} a_{4} & a_{1} & a_{1} a_{6} \\ a_{2} & & & a_{2} a_{3} & a_{4} & a_{2} a_{5} & a_{2} a_{6} \\ a_{3} & & & & a_{4} & a_{3} a_{5} & a_{6} \\ a_{4} & & & & & a_{4} a_{5} & a_{4} a_{6} \\ a_{5} & & & & & & a_{6}\end{array}$

The table defines the relations $R$ and $O$. It is then easy to extend $g$ to all of $\mathcal{P}(X)$ so that it satisfies Axioms 1 and 2.

Notice next that $f_{1}=\left(a_{1} a_{3}, a_{2} a_{3}\right), f_{2}=\left(a_{3} a_{2}, a_{6} a_{2}\right), f_{3}=\left(a_{6} a_{2}, a_{5} a_{2}\right), f_{4}=\left(a_{2} a_{5}, a_{4} a_{5}\right)$, $f_{5}=\left(a_{4} a_{5}, a_{3} a_{5}\right)$, and $f_{6}=\left(a_{5} a_{3}, a_{1} a_{3}\right)$ are edges of the graph $F$ such that $f_{1}, f_{2}$ are dichromatic, $f_{2}, f_{3}$ are monochromatic, $f_{3}, f_{4}$ are dichromatic, $f_{4}, f_{5}$ are monochromatic, $f_{5}, f_{6}$ are dichromatic, and $f_{6}, f_{1}$ are monochromatic. Then $f_{1} f_{2} f_{3} f_{4} f_{5} f_{6} f_{1}$ is a cycle in $F$ which has an odd number (three) of dichromatic pairs. See Figure 3, in which the edges of $G$ are drawn with continuous lines and the edges of $F$ are drawn as dotted lines. In 


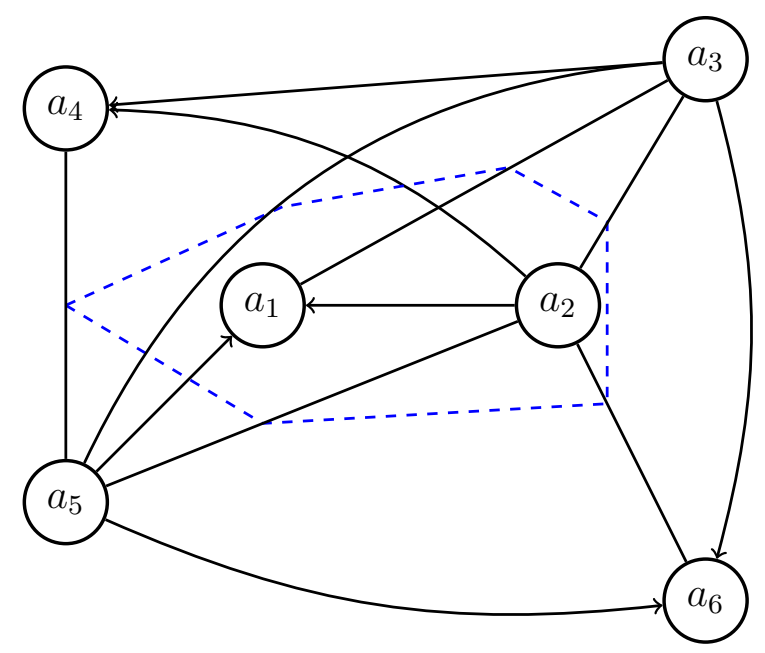

Figure 3: A choice function satisfying Axioms 1 and 2 but not 3.

Figure 3, an arrow at the end of an edge indicates the direction of $R$. The absence of any edge or arrow indicates that the edge corresponds to $O$.

The choice function $g$ then satisfies Axioms 1 and 2, but not Axiom 3 .

\section{Main results}

Our main result is a characterization of Pareto rationalizability. We express the characterization as a graph coloring problem in Theorem 8. We present the same characterization in Theorem 10, in terms of a system of quadratic equations having a solution. The version of the result in Theorem 10 may be computationally the most convenient.

In Section 3, we reduced the problem of Pareto rationalization to a problem of bicoloring the graph $F$. Our characterization of Pareto rationalization requires paying special attention to certain configurations of edges in $F$.

We say that a triple of edges $\left(f_{1}, f_{2}, f_{3}\right)$ of the graph $F$ is 3 -cyclic provided

$$
f_{1}=(c a, e a), f_{2}=(c b, e b), f_{3}=(b a, d a),
$$

for some distinct alternatives $a, b, c, d, e \in X$ with $d O e, d O c$. Notice that $\left(f_{1},(c d, e d), f_{3}\right)$, $\left(f_{3},(b c . d c), f_{1}\right)$ and $\left(f_{3},(b e, d e), f_{1}\right)$ are 3 -cyclic triples as well and that the ordering of the edges in a 3 -cyclic triple matters.

The role of 3-cyclic edges becomes apparent in the following

Theorem 8. A choice function is Pareto rationalizable if and only if it satisfies Axioms 13 , and there is a coloring of the edges of the graph F (in 1 and -1) such that two edges in a monochromatic pair have the same color, two edges in a dichromatic pair have different 


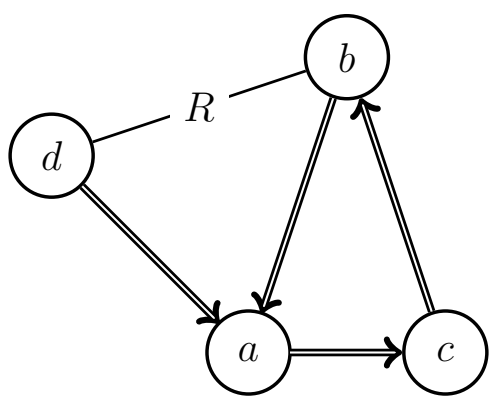

Figure 4: A cycle for $>_{1}$, indicated with directed edges.

colors, and for every 3-cyclic triple $\left(f_{1}, f_{2}, f_{3}\right)$ either $f_{1}$ and $f_{2}$ have the same color or $f_{1}$ and $f_{3}$ have the same color.

Proof. Let $g$ be a choice function that satisfies the hypotheses. We are going to construct a Pareto rationalization $\left(>_{1},>_{2}\right)$ of $g$. The construction starts by defining the two binary relations $>_{1}$ and $>_{2}$ over only some pairs of alternatives from $X$ at first, and then extending them to the entire set $X$. For each pair of alternatives $(a, b)$ with $a R b$, define $a>_{1} b$ and $a>_{2} b$. For each pair $(a, b)$ define $a>_{1} b, b>_{2} a$ if for some $c \in X$ it is the case that $(a b, c b) \in F$ is colored 1 or $(b a, c a) \in F$ is colored -1 . The pair $\left(>_{1},>_{2}\right)$ is currently only partially defined.

We present a trivial fact that will be invoked often in the sequel:

Lemma 9. Let $x, y, z \in X$ be three distinct alternatives. If $x>_{1} y>_{1} z$ and $x O y O z$, then $x O z$.

We illustrate the rest of the argument using Figures 4, 5 and 6. Suppose there is a $>_{1}$-3-cycle $a>_{1} b>_{1} c>_{1} a$ for some $a, b, c \in X$. It is easy to see that we must have a $O$ bO c $O a$ : if all three of the revealed relations belong to $R$ then $R$ is not acyclic, a contradiction; if exactly two of the relations belong to $R$ then the third must also since $R$ is transitive, a contradiction; if only one of the relations belongs to $R$ then we have not colored each edge of the graph $F$.

We represent the situation in Figure 4; we omit the label on $O$ edges to simplify the figures. The bold edges with arrows indicate the preference $>_{1}$; for example, the preference $a>_{1} b$ mandates an arrow $\Longrightarrow$ on the $a b$ edge pointing in the direction of $a$.

Now, $a O b$ and $a>_{1} b$ implies that there is $d \in X$ such that either $(b a, d a) \in F$ or $(a b, d b) \in F$. Suppose, without loss of generality, that $(b a, d a) \in F$ is the case. Then $a O d$ and $a>_{1} d$ as $(b a, d a)$ is colored -1 ; see Figure 4. Now consider the edge $c d$. By Lemma 9 we must have $c O d$. Hence $(b c, d c)$ must be an edge in $F$ colored in 1 . This means that $d>_{1} c$. (We have assumed that $(b a, d a) \in F$, but if we instead assume that $(a b, d b) \in F$, we end up with the same picture after relabeling $a, b$ and $c$.) 


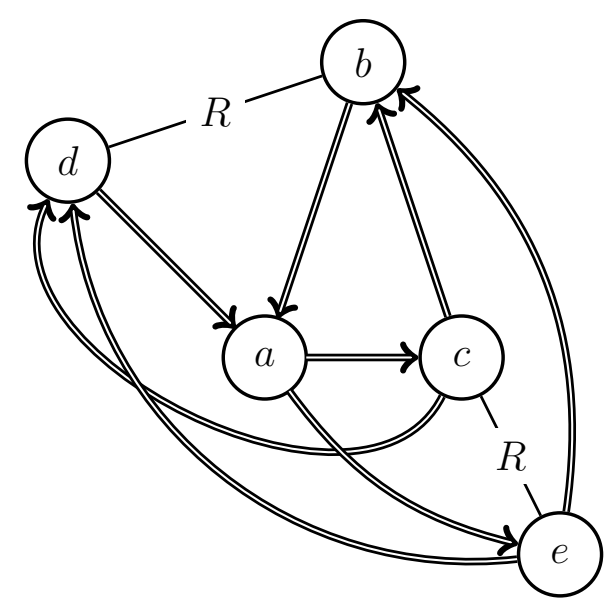

Figure 5: Implied existence of $d$ and $e$ driving the cycle.

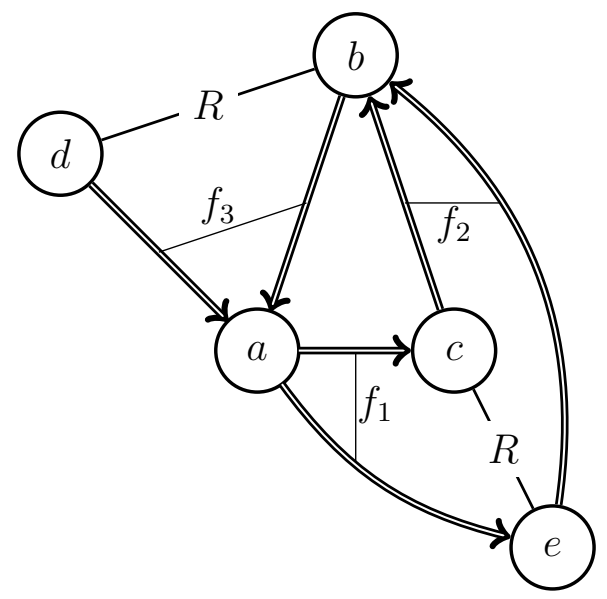

Figure 6: $f_{1}=(c a, e a), f_{2}=(c b, e b)$ and $f_{3}=(b a, d a)$.

Next, $c O a$ and $c>_{1} a$ imply the existence of some some $e \in X$ such that either $(c a, e a) \in F$ or $(a c, e c) \in F$. Suppose without loss of generality that $f_{1}=(c a, e a) \in F$. Then $e O a$ and $e>_{1} a$, as $(c a, e a)$ is colored with 1. See Figure 5 .

Note that $(c a, e a) \in F$ implies that $c R e$ or $e R c$. We invoke Lemma 9 with $e O a$, $a O b, e>_{1} a$, and $a>_{1} b$ to get $e O b$ and $b O e$. Hence, $f_{2}=(c b, e b) \in F$; in addition, $b>_{1} c$ implies that $f_{2}$ is colored -1 and thus $b>_{1} e$. See Figure 5. Now, $d O e$ as an $R$ relation is not possible by Lemma 9 . Then, $(d R b) \vee(b R d))$ implies that $(d e, b e) \in F$, and $b>_{1} e$ implies that $(d e, b e)$ is colored 1 . Hence, $d>_{1} e$. The case $(a c, e c) \in F$ leads to the same picture after relabeling $a, b$ and $c$.

Consider the edges $f_{1}=(c a, e a), f_{2}=(c b, e b)$ and $f_{3}=(b a, d a)$ which all belong to the graph $F$. Notice that $f_{3}$ colored $-1, f_{2}$ colored -1 , and $f_{1}$ colored 1 . In addition, we have $d O e, d O c$. This means that the triple of edges $\left(f_{1}, f_{2}, f_{3}\right)$ is 3 -cyclic with $f_{1}$ and $f_{2}$ having opposite colors, and $f_{1}$ and $f_{3}$ having opposite colors. We have reached a 
contradiction with the assumptions of the Lemma. Hence, there is no 3-cycle belonging to the (not yet total) relation $>_{1}$. The situation is represented in Figure 6 .

Suppose there is a 3-cycle $a>_{2} b>_{2} c>_{2} a$ which belongs to $>_{2}$. As for $>_{1}$, it must be the case that $a O b O c O a$. But then $a>_{1} c>_{1} b>_{1} a$, a contradiction.

Suppose next that $>_{1}$ is not acyclic: for some $a_{1}, a_{2}, \ldots, a_{t} \in X, t>4$ we have $a_{1}>_{1} a_{2}>_{1} \ldots>_{1} a_{t}>_{1} a_{1}$. We may assume that this is the shortest cycle, meaning that $t$ is minimal. If $a_{1} R a_{3}$ then there is a shorter cycle $a_{1}, a_{3}, a_{4}, \ldots, a_{t}, a_{1}$, a contradiction; if $a_{3} R a_{1}$, then there is a 3-cycle belonging to $>_{1}$, again a contradiction. If $a_{3} R a_{1}$ then there is a 3 -cycle that belongs to $>_{1}$, a contradiction. Hence, $a_{1} O a_{3}$. Now suppose that $a_{1} R a_{2}$. If $a_{2} R a_{3}$ then $a_{1} R a_{3}$ as $R$ is transitive, a contradiction. Then $a_{2} O a_{3}$ and $\left(a_{1} a_{3}, a_{2} a_{3}\right)$ must be an edge of the graph $F$ that is colored in 1 . We conclude that $a_{1}>_{1} a_{3}$ and there is a shorter cycle, a contradiction. So $a_{1} O a_{2}$.

This allows us to conclude that $a_{1} O a_{2} O \ldots O a_{t} O a_{1}$. Because $a_{1} O a_{2}$ and $a_{1}>_{1} a_{2}$, for some $b \in X$ either $\left(a_{2} a_{1}, b a_{1}\right) \in F$ or $\left(a_{1} a_{2}, b a_{2}\right) \in F$. Suppose that $\left(a_{2} a_{1}, b a_{1}\right) \in F$. Then $a_{1}>_{1} b$. Now $a_{t}>_{1} a_{1}, a_{1}>_{1} a_{2}$ and $a_{1}>_{1} b$ imply that $a_{t} O a_{2}$ and $a_{t} O b$, by Lemma 9 . So $\left(a_{2} a_{t}, b a_{t}\right)$ is an edge in $F$. If it is colored in -1 we get a 3 -cycle $a_{1}>_{1} a_{2}>_{1} a_{t}>_{1} a_{1}$, a contradiction. It should be colored in 1 , so $a_{t}>_{1} a_{2}$, and we once again have a shorter cycle, a contradiction. The case $\left(a_{1} a_{2}, b a_{2}\right) \in F$ leads to a contradiction in a similar fashion.

Therefore, $>_{1}$ is acyclic and can be extended to a total, antisymmetric and transitive relation (a preference relation) on $X$. In light of observation (2) in Section 3, the (extended) preference relation $>_{1}$ extends $>_{2}$ to a total and antisymmetric binary relation on $X$ which does not possess any 3 -cycles. On the other hand, if a total relation has a cycle, it is easy to see that it has a 3-cycle. It follows that $>_{2}$ is also acyclic and Lemma 4 implies that $\left(>_{1},>_{2}\right)$ is a rationalization of the choice function $g$.

We finish the proof by proving the converse statement. Let $\left(>_{1},>_{2}\right)$ be a rationalization of the choice function $g$. We have already shown that $g$ must satisfy Axioms 1- 3 . We have also seen in Lemma 6 that the rationalization $\left(>_{1},>_{2}\right)$ induces a coloring of the edges of the graph $F$ that respects di- and monochromatic pairs. Finally, if $\left(f_{1}, f_{2}, f_{3}\right)$ is a 3-cyclic triple and we suppose that $f_{1}$ and $f_{2}$ have opposite colors and $f_{1}$ and $f_{3}$ have opposite colors, we must conclude that either $a>_{1} b>_{1} c>_{1} a$ or $a>_{1} c>_{1} b>_{1} a$. A contradiction of the transitivity of $>_{1}$. Hence, $f_{1}$ and $f_{2}$ have the same color or $f_{1}$ and $f_{3}$ have the same color.

Remark. The proof of Theorem 8 is constructive. Given a coloring of the edges of the graph $F$, we described a simple procedure for constructing a rationalization $\left(>_{1},>_{2}\right)$ of the given choice function: the rationalization must agree with the revealed relation $R$, as well as the coloring of the graph $F$ over pairs of alternatives $a b$ which represent an endpoint of an edge in the graph $F$. Next, we extend $>_{1}$ to the entire set $X$, which also determines $>_{2}$ as a linear order on $X$. We showed that the pair $\left(>_{1},>_{2}\right)$ constructed in this way is indeed a rationalization. 
Theorem 8 is a characterization of Pareto rationalizability. From the results in Section 3, we know that, if a choice function satisfies Axioms 1-3 there is a coloring of the edges of the induced graph $F$ which assigns opposite colors to edges from a dichromatic pair and equal colors to edges from a monochromatic pair. Pareto rationalizability amounts, over and above Axioms 1-3, to finding a coloring of the edges of $F$, which in addition to respecting di- and monochromatic pairs also has the property that for every 3 -cyclic triple $\left(f_{1}, f_{2}, f_{3}\right)$ either $f_{1}$ and $f_{2}$ have the same color or $f_{1}$ and $f_{3}$ have the same color.

Notice that if there is a coloring of the edges of $F$ that satisfies the conditions in Theorem 8, we can reverse the color of every edge and still have a coloring that satisfies those conditions: the reversed coloring leads to the same rationalizing pair as the original one, with $>_{2}$ in place of $>_{1}$. To avoid dealing with this duality, and to simply our notation, we introduce a third graph $H$. The vertexes of $H$ are the edges of the graph $F$, and two vertexes $f_{1}, f_{2}$ in $H$ are joined by an edge in $H$ if and only if there is a path in $F$ connecting $f_{1}$ with $f_{2}$.

In the graph $F$, the notions of monochromatic or dichromatic pairs of edges only applied to adjacent edges; that is, edges with a common vertex. We can easily extend this definition to pairs of edges that are connected by a path in $F$, calling a pair of edges monochromatic if there is a path with an even number of dichromatic pairs joining them, and dichromatic if there is a path with an odd number of dichromatic pairs joining them. The more general notion of mono- and di-chromatic is well-defined under Axiom 3, as Axiom 3 then insures that no pair of edges from $F$ is both dichromatic and monochromatic.

Under Axiom 3, then, we can extend our definitions of dichromatic and monochromatic edges in $F$ to give a bi-coloring of $H$. We say that the edge $f_{1} f_{2}$ (in $H$ ) is colored 1 if the corresponding path (in $F$ ) has an even number of dichromatic pairs, and is colored -1 if the path has an odd number of dichromatic pairs.

Now, $H$ is the dual graph of $F$. It is well-known and easy to see that Axiom 3 is equivalent to

Axiom 3'. Every cycle in $H$ has an even number of edges colored -1 .

Observe that a choice function induces a coloring of the edges in $H$ just like it labels some pairs of edges in $F$ as di- or mono-chromatic. We cannot choose the coloring of the edges of $H$, in contrast to the coloring of $F$ we have discussed above.

We now express the consequences of Theorem 8 for $H$ using a system of equations. A solution to the system of equations will be a coloring of the vertexes of $H$.

For the graph $H$, Theorem 8 implies the following. A choice function that satisfies Axioms 1-3 is Pareto rationalizable if and only if there is a coloring of the vertexes of $H$ in 1 and -1 , such that 
- two vertexes $h_{1}, h_{2} \in H$, such that $h_{1} h_{2}$ is and edge in $H$, have equal colors if $h_{1} h_{2}$ is colored 1 and opposite colors if $h_{1} h_{2}$ is colored -1 ;

- for every 3-cyclic triple of vertexes $\left(h_{1}, h_{2}, h_{3}\right)$ either $h_{1}$ and $h_{2}$ have the same color or $h_{1}$ and $h_{3}$ have the same color. ${ }^{5}$

We introduce one variable for each vertex of $H$; these variables can take the values 1 or -1 .

Let $k$ be the number of 3-cyclic triples:

$$
T_{1}=\left(h_{1,1}, h_{1,2}, h_{1,3}\right), T_{2}=\left(h_{2,1}, h_{2,2}, h_{2,3}\right), \ldots, T_{k}=\left(h_{k, 1}, h_{k, 2}, h_{k, 3}\right) .
$$

Remark. As we observed before, 3-cyclic triples come in groups of four. It is sufficient to satisfy the requirement in Theorem 8 only about one 3-cyclic triple out of those four and we may choose to ignore the other three. For theoretical purposes, the redundancy plays no role. For computationally constructing a Pareto rationalization, it would be important to only include one representative 3-cyclic triple.

We proceed to describe a system of equations, named $(*)$, by enumerating all the component equations.

First, include the $3 k$ equations

$$
h_{i, j}^{2}=1
$$

in the system $\left(^{*}\right)$. These must be included because $h_{i, j}$ must take the value 1 or -1 .

Next, if the same vertex belongs to two different 3-cyclic triples, we have that

$$
h_{i_{1}, j_{1}}=h_{i_{2}, j_{2}}
$$

belongs to $\left(^{*}\right)$, where $h_{i_{1}, j_{1}}$ and $h_{i_{2}, j_{2}}$ are the same vertex in $H$. If $h_{i_{1}, j_{1}}$ and $h_{i_{2}, j_{2}}$ are two vertexes for which $h_{i_{1}, j_{1}} h_{i_{2}, j_{2}}$ is an edge in $H$ colored 1 , then

$$
h_{i_{1}, j_{1}}=h_{i_{2}, j_{2}}
$$

is an equation in the system; if $h_{i_{1}, j_{1}} h_{i_{2}, j_{2}}$ is an edge in $H$ colored -1 , then

$$
h_{i_{1}, j_{1}}=-h_{i_{2}, j_{2}}
$$

must be included in the system of equations $(*)$.

Finally, for every $i=1,2, \ldots, k$, the equation

$$
\left(h_{i, 1} h_{i, 2}+h_{i, 1} h_{i, 3}-1\right)^{2}=1
$$

belongs to the system $\left(^{*}\right)$. Notice that $h_{i, 1}$ and $h_{i, 2}$ having opposite colors or $h_{i, 1}$ and $h_{i, 3}$ having opposite colors is equivalent to $h_{i, 1} h_{i, 2}+h_{i, 1} h_{i, 3} \neq-2$, which is equivalent to $h_{i, 1} h_{i, 2}+h_{i, 1} h_{i, 3} \in\{0,2\}$. This, in turn, is equivalent to $\left(h_{i, 1} h_{i, 2}+h_{i, 1} h_{i, 3}-1\right)^{2}=1$.

\footnotetext{
${ }^{5} \mathrm{~A}$ triple of vertexes $\left(h_{1}, h_{2}, h_{3}\right)$ in $H$ is called 3 -cyclic if and only if that same triple viewed as a triple of edges in $F$, is 3 -cyclic.
} 
Axiom 4. The system of equations (*) has a solution.

Evidently, Axiom 4 is simply a reformulation of the conditions in Theorem 8. So Axiom 4 is both a necessary and sufficient condition for the existence of a coloring of the vertexes of $H$ in the hypotheses of Theorem 8. We thus obtain:

Theorem 10. The choice function $f$ is Pareto rationalizable if and only if it satisfies Axioms 1,2,3,4.

Unfortunately, the system $\left(^{*}\right)$ is not linear, and its solution may present significant computational problems. That said, the construction of the system should be computationally feasible (all the graphs involved can be constructed in polynomial time). One computational advantage of the system is that it does not involve equations of order higher than 2 , as $h_{i, j}^{2}=1$ implies that

$$
\left(h_{i, 1} h_{i, 2}+h_{i, 1} h_{i, 3}-1\right)^{2}=3+2 h_{i, 2} h_{i, 3}-2 h_{i, 1} h_{i, 2}-2 h_{i, 1} h_{i, 3} .
$$

\section{Rationalizability in non-strict preferences}

We proceed to discuss rationalization by non-strict preferences. We observe that our characterization still holds in this case, when we define Pareto efficiency in the weak sense that an alternative $a$ in $B$ is Pareto efficient if there is no $b \in B$ that both agents strictly prefer.

A choice function $g$ is two-agent Pareto rationalizable in non-strict preferences if there are two total, reflexive and transitive binary relations $>_{1}$ and $>_{2}$ on the universal set of alternatives $X$, such that for all $B \in \mathcal{P}(X), f(B)$ is the set of Pareto efficient alternatives in $B$ with respect to $\left(>_{1},>_{2}\right)$. In this case, we say that $\left(>_{1},>_{2}\right)$ is a Pareto rationalization of $g$ in non-strict preferences.

Theorem 11. The choice function $g$ is Pareto rationalizable in non-strict preferences if and only if it satisfies Axioms 1,2,3,4.

Proof. Suppose $g$ satisfies Axioms 1 - 4. Then Theorem 10 implies that $g$ is rationalizable by some strict preferences $\left(>_{1},>_{2}\right)$, and therefore $g$ is also rationalizable by non-strict preferences. On the other hand, suppose $g$ is rationalizable by nonstrict $\left(>_{1},>_{2}\right)$. It is readily seen that axioms 1-2 must still hold. Also, if $a R b, b O c, c O a$, then $>_{1}$ and $>_{2}$ must be strict on $(a, b)$ and $(b, c)$ : if $b={ }_{1} c$ then $b={ }_{2} c$ and it must be the case that $a R c$ since $a R b$. This contradicts $a O c$. The remaining axioms 3,4 only deal with these tractable pairs $b O c$ and $c O a$, over which there are can be no indifferences belonging to rationalizing relations, and it follows that they are also necessary. 


\section{Conclusion}

We characterize two-agent Pareto rationalizability of choice functions. We present several simple tests for Pareto rationalizability, most notably the verification of Axiom 3. This axiom is a new consequence of Pareto rationalizability, and its verification seems to be computationally simple.

Our characterization, on the other hand, essentially involves solving a system of quadratic equations. Thus, applying our characterization may be computationally hard; it may not present a substantial advantage over exhaustively searching over all possible rationalizing linear orderings. For problems of a given size, though, it seems intuitive that the constructions we have discovered (the graph $H$ ) simplifies the problem.

Finally, we have worked on the two-agent case. We do not know if our graph-theoretic approach extends to the case when we have more than two agents. The difficulty is that there is no two-coloring which allows us to formulate meaningful, simple graph tests, which seem to lead in the direction of a characterization. The general problem with $n$ agents remains open.

\section{References}

Dushnik, B., And E. W. Miller (1941): "Partially Ordered Sets," American Journal of Mathematics, 63(3), 600-610.

Moulin, H. (1991): Axioms of Cooperative Decision Making. Cambridge University Press, Cambridge, UK.

Sprumont, Y. (2000): "On the Testable Implocations of Collective Choice Theories," Journal of Economic Theory, 93, 205-232.

(2001): "Paretian Quasi-orders: The Regular Two-Agent Case," Journal of Economic Theory, 101(2), 437 - 456.

Trotter, W. T. (2001): Combinatorics and Partially Ordered Sets: Dimension Theory. Johns Hopkins University Press, Baltimore, MD. 